\title{
Factors Influencing Automated Limited Lead Detection of Atrial Fibrillation
}

\author{
Peter Macfarlane $^{1}$, Shahid Latif ${ }^{1,}$ Brian Devine ${ }^{1}$ \\ ${ }^{1}$ Institute of Health and Wellbeing, University of Glasgow
}

\begin{abstract}
There has been interest relating to automated analysis of a lead I ECG to detect cardiac arrhythmias. Little interest has been shown in the accuracy of using lead I as opposed to 6 limb leads or the full 12 lead ECG. The aim of this small study was to assess the efficacy of using only lead I but also to look at the effect of analysing a single 30 s recording as a continuous recording versus five $10 \mathrm{~s}$ overlapping recordings constituting a 30s record.

One hundred 10s digital 12 lead ECGs with atrial fibrillation $(A F)$ were used. Chest leads were removed and the 6 limb leads then used for analysis of rhythm. Similarly, lead I alone was used. Separately 100 single lead I ECGs classified as AF in the PhysioNet 2017 database were analysed, both as single 30s recordings and as five 10s ECGs commencing at 0, 5, 10, 15 and 20s from the start of the recording. An algorithm made the diagnosis from 5 reports. All analyses were made with the Glasgow Program. For the 10s 12 lead ECGs, 96\% were reported as AF using 6 limb leads and 93\% using lead I. For the 30s recordings, 92\% were reported as AF using a single 30 s analysis and $91 \%$ as AF using the five ECGs.

In conclusion, one lead and 6 leads are not as sensitive as 12 leads in detecting $A F$, while five 10 s reports combined are no more sensitive than a single 30s report though more specific.
\end{abstract}

\section{Introduction}

\subsection{Aim}

In recent years, there has been considerable interest in the use of a single lead short term ECG recording for the detection of cardiac arrhythmias and in particular, atrial fibrillation (AF). This follows from the introduction of the AliveCor device [1] whereby a small two electrode array is used to record lead I with a wireless link to a mobile telephone to analyse the ECG. The approach was transferred to a wrist watch for research purposes where the left arm potential was recorded directly from the back of the watch and the right arm potential was recorded from the right hand touching an electrode on the strap.

More recently, Apple introduced the Apple 4 watch which similarly can interpret a single lead I ECG recording where one electrode is attached to the back surface of the watch touching the left wrist and the other electrode is the crown of the watch on which a finger from the right hand is placed [1]. The watch can be worn on the right wrist with arm connections reversed.

The PhysioNet 2017 Challenge was actually based on a large dataset of ECGs recorded with the AliveCor device and many different approaches were used for analysing the single channel ECG. A number of manuscripts from the competition are available in the 2017 Computing in Cardiology Proceedings [2]. In that study, ECGs were classified into four groups, namely normal, AF, other arrhythmia and noisy. A number of the groups in the competition had results involving F1 as a measure with values exceeding 0.8 . This represents a form of average measure for sensitivity and positive predictive value from each of the four categories.

However, while AF may be defined on the basis of an irregular RR interval, it can be the case that in lead I there is no clear atrial activity exhibited. Thus, for example in the case of one short RR interval in a short recording, there is certainly an irregularity but without any evidence of atrial activity in lead I, this could be possible AF or it could be sinus rhythm with a premature atrial complex.

It was therefore thought of interest to assess the effect on accuracy of detecting AF by reducing the number of leads from 12 to 1 , with the selected lead being lead I because of its use in the recently introduced devices.

It was also thought to be of interest to look at the recording of leads I and II from which the remaining limb leads can be derived to see if this was better than recording a single lead ECG. Some preliminary work had been done in this laboratory previously [3].

As a separate study, it was also of interest to see how the 10s analysis of the Glasgow Program could be adapted for analysis of the 30s single lead recordings from the PhysioNet 2017 Challenge database. To this end, two possibilities arose. One was to adjust the amount of data which could be analysed by the Glasgow Program to handle 30s of a single lead and the other was to divide the 30 s recording into five 10 s recordings spaced at 5 second intervals from the start of the recording.

\section{Methods}

A consecutive batch of 12 lead ECGs with a confirmed 
diagnosis of AF, as reported by the Glasgow Program [4] was selected from a local database. ECGs were sampled at 500 samples per second and the duration of each recording was 10 s. In order to create a 6 lead ECG, the precordial leads V1-V6 were set as unavailable. Similarly, to create a single lead ECG, all leads except lead I were set as unavailable.

In order to analyse a single lead ECG, missing leads from the 12 lead ECG were created from the single lead. For example, lead V1 was set as an inverted lead I. Lead V6 was set as lead I. Thus, in the case of the single lead ECG, each of the missing 11 leads was a function of lead I. Similarly, for the 6 lead analysis, the missing precordial leads were also set as a function of lead I.

Thus, the standard 12 lead ECG analysis software incorporated into the Glasgow Program could be used to analyse a single lead ECG or a 6 lead ECG of 10 seconds duration.

For the second part of the study, the single lead data from the PhysioNet 2017 Challenge dataset was used. The duration of these leads varied from under 30s to well in excess of 30s but for this study, only those ECGs which lasted at least 30s were used. Consecutive single lead I ECGs, classified as AF in the PhysioNet 2017 Challenge Training Set, were selected. On this occasion, these ECGs were also processed in two different ways.

\section{1 $\quad$ Method 1}

The 30s single lead ECG was divided into five $10 \mathrm{~s}$ segments with the onset of the segments being at the start of the recording, 5, 10, 15 and 20 s after the start of the recording. Thus, the 30 second recording was split in to 5 recordings of 10 s each.

These single lead recordings were then transformed into 12 lead recordings as previously described. These 12 lead recordings were then processed by the Glasgow Program giving five rhythm interpretations.

An algorithm was then used to combine the rhythms from the five reports. For example, if three interpretations said AF and two said sinus rhythm with supraventricular extrasystoles, then the final report would have been AF.

\subsection{Method 2}

The software for analysis of rhythm within the Glasgow Program was extended to cope with 30 seconds of data, simply by increasing the matrix size for the data files. The actual diagnostic logic of the rhythm program was not altered in any way for this pilot study.

Thus, the single lead 30s ECG became a 12 lead ECG with a duration of 30s and the Glasgow Program was used to interpret the rhythm in the usual way. This was feasible because various indices of regularity, whether it be for the $\mathrm{RR}$ interval or the PR interval were expressed as percentages within the logic.

\section{Results}

\subsection{Limited Lead Analysis}

The 10012 lead ECGs with a diagnosis of AF were analysed by the 6 lead and 1 lead versions of the software. Table 1 shows the results. Clearly, reducing the number of leads resulted in a decreased sensitivity for detecting AF with $96 \%$ for the 6 lead data and $93 \%$ for the single lead data.

\begin{tabular}{|l|c|c|c|}
\hline Type of ECG & Definite AF & Probable AF & Total \\
\hline 12-lead & 100 & 0 & 100 \\
\hline 6-lead & 95 & 1 & 96 \\
\hline 1-lead & 91 & 2 & 93 \\
\hline
\end{tabular}

Table 1: Sensitivity of detecting AF from 10s recordings with different leads.

\subsection{PhysioNet 2017 Database}

A continuous set of ECGs was reviewed until 100 cases regarded as $\mathrm{AF}$ according to the database classification were available. That resulted in a total of 1074 ECGs being analysed with 974 ECGs having been classified as normal, other or noisy according to the PhysioNet data base.

Results based on using the approach of five separate $10 \mathrm{~s}$ reports with a single resultant diagnosis, as well as a single analysis of 30s recordings, are provided in Table 2 .

\begin{tabular}{|l|c|c|}
\hline Type of ECG & Sensitivity & Specificity \\
\hline $5 \times 10 \mathrm{~s}$ & $91.0 \%$ & $85.4 \%$ \\
\hline $30 \mathrm{~s}$ & $92.0 \%$ & $76.3 \%$ \\
\hline $10 \mathrm{~s}$ and $30 \mathrm{~s}$ & $86.0 \%$ & $90.5 \%$ \\
\hline $10 \mathrm{~s}$ or $30 \mathrm{~s}$ & $97.0 \%$ & $71.3 \%$ \\
\hline
\end{tabular}

Table 2: Sensitivity and specificity of detecting AF from 30 s recordings using different approaches.

Using the single lead 30 second approach resulted in a sensitivity of $92 \%$ but a reduced specificity as shown in Table 2.

In this part of the study, there was local disagreement with the PhysioNet classification in 2/8 and 4/9 of the false negative AF reports. Effectively, this means that there were several cases where AF was regarded as the incorrect classification of an ECG and was reported as such by the Glasgow Program. 


\section{Discussion}

While a great deal of attention has been paid to the accuracy of detecting AF using the single channel ECG, not much discussion has related to the fact that the use of a single lead in itself reduces sensitivity, as evidenced by this study. The reduction determined in this study was up to $7 \%$ which is a modest percentage. The 12 lead ECGs used for this part of the study were of reasonable quality and all had been reported as AF initially. Thus there was no suggestion of noise interfering with the interpretation.

It was thought that, by dividing the 30 s recording into five different segments, short bursts of noise would affect only one or two of the interpretations, leaving the others to produce a correct interpretation. It appears that this did not work as well as had been hoped although the number of false positive reports of $\mathrm{AF}$ and Other were much higher using the single 30 s analysis compared to the five 10 s segmented analyses. In large part, this can be attributed to noisy sections of recording, leading to false positive detections of QRS complexes and hence, irregular rhythm.

It is instructive to consider some examples from the database and the results from the different approaches to analysis.

Figure 1 shows an example of ectopic atrial bradycardia where there is a prominent $\mathrm{P}$ wave in lead I which would undoubtedly be reported as sinus rhythm in a single lead analysis.

Figure 2 illustrates 510 s segments in a 30s recording where there is noise at around 16s. This is sufficient to result in a report of Sinus rhythm with PVCs. However, if this ECG is analysed as $510 \mathrm{~s}$ recordings, then 3 are reported as Sinus rhythm and 2 as Other, i.e. Sinus with PVCs and so the result is Sinus Rhythm.

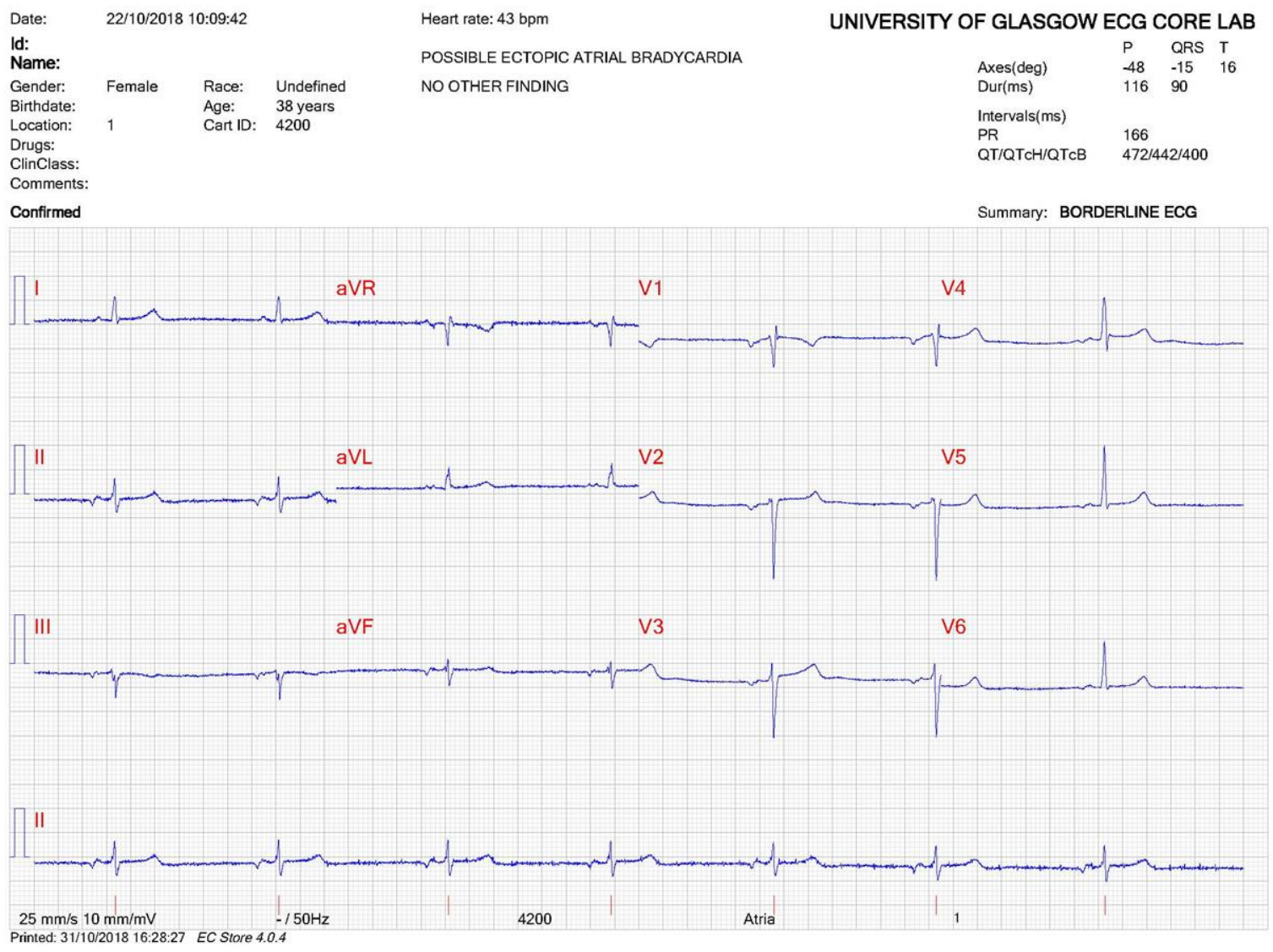

Figure 1. Ectopic atrial bradycardia evidenced by an inverted $\mathrm{P}$ wave in III, aVF and a $\mathrm{P}$ axis of $-48^{\circ}$. 


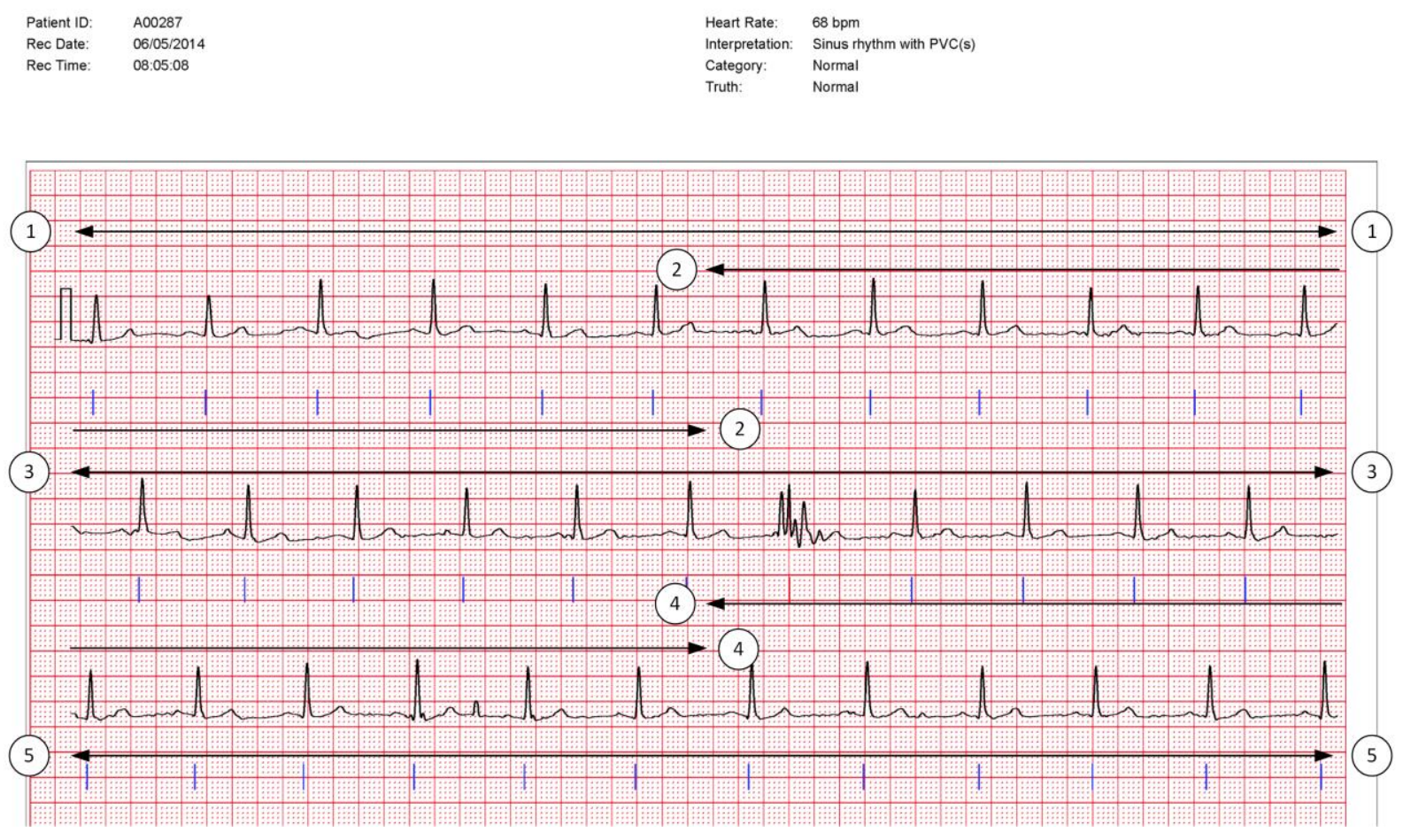

Figure 2. 30s single lead recording divided into 5 segments each of 10s labelled 1 - 5. Noise can be seen at around 16s.

The 30s single lead analysis uses the basic Glasgow Program. It is possible that one or two fixed criteria within the logic would have been less meaningful when 30s were used compared to the original 10s analyses. However, as previously mentioned, the vast majority of criteria related to percentage values of irregularity etc. This study did not allow for a detailed review of the logic to check every single criterion and so the possibility remains that $30 \mathrm{~s}$ single lead analysis could be enhanced to a limited extent with a further review of the basic rhythm interpretation.

By far the biggest problem in all of these recordings remains noise. Further modification of the five separate analyses could be made by careful assessment of noise and omission of a $10 \mathrm{~s}$ segment which included significant noise, hopefully leaving two or three 10s segments with little or no noise on which to make an interpretation.

This study was preliminary in the sense that existing software was used for exploratory purposes. What remains to be done is an assessment of how to handle noise and to consider whether any criteria in the analysis have to be adjusted in a 30 s recording.

It was noted that at least one publication from work in the PhysioNet 2017 Challenge finessed the question of noise in order to obtain satisfactory results.

The overall conclusion is that a single lead will result in approximately a $7 \%$ loss in the detection of AF compared the use of a 12 lead ECG. Multiple 10s analyses in a 30s recording are equally sensitive to analysis of a single $30 \mathrm{~s}$ recording but more specific. However, the logic for the $30 \mathrm{~s}$ analysis was designed for a 10 s recording and requires to be reviewed.

\section{References}

[1] Bennett R, French A. Rise of the smart device ECG and what it means for the general cardiologist.

doi:10.1136/heartjnl-2019-315357

[2] Clifford G, Liu C, Moody B et al. AF Classification from a Short Single Lead ECG Recording: the PhysioNet/Computing in Cardiology Challenge 2017. doi:10.22489/CinC.2017.065-469

[3] Griffiths K, Clark EN, Devine B, Macfarlane PW. Assessing the Accuracy of Limited Lead Recordings for the Detection of Atrial Fibrillation. Computing in Cardiology 2014; 41:405-408.

[4] Macfarlane PW, Devine B, Clark E. The University of Glasgow (Uni-G) ECG Analysis Program. Computing in Cardiology 2005;32:451-454.

Address for Correspondence:

Professor Peter Macfarlane

Electrocardiology Lab, New Lister Building

Royal Infirmary, 10 Alexandra Parade

Glasgow G31 2ER, Scotland.

e-mail:peter.macfarlane@glasgow.ac.uk 